OPEN ACCESS

Edited by:

Shourong Shi,

Poultry Institute, Chinese Academy of Agricultural Sciences (CAAS), China

Reviewed by: Jiangchao Zhao,

University of Arkansas, United States

Yingping Xiao

Zhejiang Academy of Agricultural

Sciences, China

${ }^{*}$ Correspondence:

Qiugang Ma

maqiugang@cau.edu.cn

Specialty section:

This article was submitted to Nutrition and Microbes,

a section of the journal

Frontiers in Nutrition

Received: 07 November 2021 Accepted: 04 January 2022

Published: 10 February 2022

Citation:

Guo Y, Huang S, Zhao L, Zhang J, Ji $C$ and $M a Q$ (2022) Pine (Pinus massoniana Lamb.) Needle Extract

Supplementation Improves

Performance, Egg Quality, Serum Parameters, and the Gut Microbiome in Laying Hens. Front. Nutr. 9:810462.

doi: 10.3389/fnut.2022.810462

\section{Pine (Pinus massoniana Lamb.) Needle Extract Supplementation Improves Performance, Egg Quality, Serum Parameters, and the Gut Microbiome in Laying Hens}

\author{
Yanxin Guo, Shimeng Huang, Lihong Zhao, Jianyun Zhang, Cheng Ji and Qiugang Ma*
}

State Key Laboratory of Animal Nutrition, College of Animal Science and Technology, China Agricultural University, Beijing, China

The effects of Masson pine (Pinus massoniana Lamb.) needle extract (PNE) on gastrointestinal disorders and oxidative stress have been widely investigated using experimental models; however, the functions and mechanisms of these effects in chicken models remain unknown. We investigated the effects of Masson PNE supplementation on performance, egg quality, serum parameters, and the gut microbiome in laying hens. A total of 60 healthy 50-week-old Peking Pink laying hens with similar body conditions and egg production were randomly divided into the control (CON) (0 mg/kg PNE), PNE100 (100 mg/kg PNE), PNE200 (200 mg/kg PNE), and PNE400 (400 mg/kg PNE) groups, with fifteen replicates per treatment and one hen per replicate. Compared with the CON group, egg mass, feed conversion ratios, and yolk weight were significantly increased (p $<0.01$ ) in the PNE100 group. Dietary supplementation of $100 \mathrm{mg} / \mathrm{kg}$ PNE increased the serum total protein, albumin, and glucose concentrations $(p<0.01)$ and decreased the alanine aminotransferase activity $(p<0.05)$ compared with those of the CONs. Hens in the PNE100 group had reduced serum malondialdehyde levels $(p<0.05)$ and increased catalase, superoxide dismutase, and glutathione peroxidase activities $(p<0.01)$ compared with those of the CON group. Serum proinflammatory cytokine concentrations of interleukin (IL)-1 $\beta, I L-6$, and tumor necrosis factor- $\alpha$ were lower $(p<0.01)$ and the $\mathrm{IL}-10$ level was higher $(p<0.01)$ in the PNE100 group than in the CON group. Serum immunoglobulin (Ig)A, IgG, and IgM concentrations were increased in the PNE100 group $(p<0.01)$. The relative abundance of Bacteroidetes was increased, while the relative abundances of Firmicutes and Proteobacteria were decreased in the PNE100 group. The relative abundances of Vibrio, Shewanella, and Lactobacillus were decreased, while the relative abundances of unclassified_o_Bacteroidales, Rikenellaceae_RC9_gut_group, unclassified_f_Rikenellaceae, and Butyricicoccaceae were increased in the PNE100 group compared with those of the CON group. PNE supplementation at $100 \mathrm{mg} / \mathrm{kg}$ improved the diversity and structure of the gut microbial composition, production performance, egg quality, and serum parameters of laying hens. The laying hens in this study had good production performance when supplemented with 100 mg/kg PNE.

Keywords: laying hens, antioxidant, immune response, gut microbiota, pine (Pinus massoniana Lamb.) needle 


\section{INTRODUCTION}

Poultry intestinal tracts possess complex microbial communities consisting of dominant bacteria that play key roles in host performance and gut health (1). Multiple factors, such as age, production system, disease, and diet, influence the gut microbiota of laying hens $(2,3)$. Additionally, diet is a major factor influencing the gut microbial community $(4,5)$. Hence, improving poultry gut health may improve final production performance to market. Many strategies (e.g., feeding modulations, acid preparations, probiotics, and enzymes) are incorporated to protect the gut from external infections and ensure that poultry have maximum potential genetic productivity (6). Phytogenic feed additives are gaining attention for their active components and compatible biological characteristics, which have been reported to benefit animal production and health (7). Masson pine (Pinus massoniana Lamb.) is a highly economic tree species containing terpenoids, phenolic compounds, flavonoids, and alkaloids (8), which inhibit peroxidation (9), promote immunity (10), and confer antibacterial properties (11). One study found that adding pine needles improved quail performance and serum antioxidant systems. Broilers-fed pine powder had reduced serum total cholesterol (12) and improved meat quality. Pine needle powder increased growth performance in broilers by improving the small intestinal length and protein digestibility (13). Moreover, pine needle extracts (PNEs) have antimicrobial and anti-inflammatory activities against pathogens, stimulating growth of beneficial gut bacteria, particularly Enterococcus spp. and Bifidobacterium spp. $(14,15)$. However, no study has adequately demonstrated the benefits of PNEs on poultry performance, especially for laying hens.

We speculated that in late egg production, laying hensfed PNE would show improved production performance, antioxidant abilities, and immune system and inflammatory responses compared with those of control (CON) diet-fed birds and that these effects would be associated with the benefits of PNE for the gut. Thus, this study was conducted to determine the appropriate amount of dietary PNE for laying hens according to its effects on production performance, egg quality, serum parameters, and the gut microbiota.

\section{MATERIALS AND METHODS}

The experimental protocols were approved by the institutional Animal Care and Use Committee of China Agricultural University (AW92111202-1-1, Beijing, China) and the methods were carried out in accordance with the relevant guidelines and regulations.

\section{Hens and Management}

Laying hens were allocated to the same tier of battery cages with one hen per cage (cage size: $40 \times 40 \times 40 \mathrm{~cm}$ ) and exposed to light $(16 \mathrm{~h} /$ day $)$ with an intensity of $16 \mathrm{~lx}$. The temperature was maintained at $18-25^{\circ} \mathrm{C}$ throughout the experiment. Food and water were offered ad libitum in mash form and by nipple drinkers, respectively. All the hens remained in good health
TABLE 1 | Ingredient and nutrient composition of gestation diets (\%).

\begin{tabular}{lc}
\hline Ingredients & Content \\
\hline Corn & 56.40 \\
Soybean meal & 25.00 \\
Corn starch & 8.00 \\
Limestone & 8.30 \\
CaHPO 4 & 1.50 \\
NaCl & 0.30 \\
DL-Methionine & 0.17 \\
Minerals premix & 0.30 \\
Vitamins premix & \\
Total & 0.03 \\
Nutrient levels & 100.00 \\
Metabolizable Energy (ME)/(MJ/kg) ${ }^{\mathrm{C}}$ & \\
CP & 11.30 \\
Lys & 15.20 \\
Met & 0.82 \\
Met + Cys & 0.40 \\
Ca & 0.64 \\
Total P & 3.56 \\
Available P & 0.54 \\
\hline & \\
\hline
\end{tabular}

$a_{1} \mathrm{~kg}$ of vitamin premix contained the following: VA 8,000 IU VD $D_{3} 3,600 \mathrm{IU}$; VE $21 \mathrm{IU} ; \mathrm{VK}_{3}$ $4.2 \mathrm{mg} ; V B_{1} 3 \mathrm{mg} ; V_{2} 10.2 \mathrm{mg}$; folic acid $0.9 \mathrm{mg}$; calcium pantothenate $15 \mathrm{mg}$; nicotinic acid $45 \mathrm{mg} ; \mathrm{VB}_{6} 5.4 \mathrm{mg} ; \mathrm{VB}_{12} 0.024 \mathrm{mg}$; and biotin $0.15 \mathrm{mg}$.

${ }^{b} 1 \mathrm{~kg}$ of mineral premix contained the following: $\mathrm{Cu} 6.8 \mathrm{mg}$; Fe $66 \mathrm{mg}$; Zn 83 mg; $\mathrm{Mn}$ $80 \mathrm{mg}$; $10.6 \mathrm{mg}$; and Se $0.3 \mathrm{mg}$.

${ }^{c} M E$ was a calculated value, while the others were measured values.

during the feeding period. No hens were culled nor any hens receive medical intervention.

\section{Experimental Design and Diets}

A total of 60 50-week-old Peking Pink hens (Yukou Poultry Corporation Ltd., Beijing, China) were randomly allocated to one of four treatments. Previous studies fed poultry $150 \mathrm{mg} / \mathrm{kg}$ PNE; thus, we used different concentrations (100, 200, and 400 $\mathrm{mg} / \mathrm{kg}$ ) of PNE to comprehensively investigate the appropriate amount of dietary PNE for laying hens according to its effects on performance, egg quality, serum parameters, and the gut microbiota. Hens were fed a corn-soybean meal-based diet (Table 1) supplemented with PNE at 0 (the CON group), 100, 200 , or $400 \mathrm{mg} / \mathrm{kg}$. Each treatment consisted of 15 replicates with one bird in 15 adjacent cages per replicate. The basal diet was formulated according to the nutrient requirements for laying hens (NY/T 33-2004), published by the Chinese Ministry of Agriculture (2004). The PNE product, containing $22.11 \mathrm{mg} / \mathrm{kg}$ flavonoids and $10.50 \mathrm{mg} / \mathrm{kg}$ shikimic acid, was purchased from Shaanxi Yunqi Biotechnology Corporation Ltd., Shaanxi, China. The entire experiment lasted 9 weeks (from 50 to 58 weeks of age) consisting of a 1-week adaptation period and an 8-week experimental period.

\section{Performance and Egg Quality Parameters}

Daily egg counts, total egg weight, and weekly feed consumption were recorded and calculated weekly as hen-day egg production, 
egg mass, average egg weight, average daily feed intake, and feed conversion ratio (FCR). Thirty eggs per treatment were collected for interior and exterior quality tests during the last 3 days of the end of week 58. Shell strength was measured with an egg force reader (ESTG-01, Orka Technology Ltd., Bountiful, UT, USA). Shell thickness was measured using a shell thickness tester (ESTG-01, Orka Technology Ltd.). Haugh unit, yolk color, and egg weight were measured using a multifunctional egg quality tester (EA-01, Orka Technology Ltd.). Shell color was measured with a QCR color reflectometer (QCR SPA, TSS, England, UK). The shells were weighed, the yolks were separated with a separator, and the yolk and albumin percentages were weighed and calculated.

\section{Blood Sample Collection and Analysis}

At the end of the experiment, blood samples were collected from the wing veins of the laying hens on the same day of sampling and centrifuged at 3,000 rpm for $15 \mathrm{~min}$ at room temperature to separate the serum. Subsequently, serum samples were collected via pipette into 1.5 - $\mathrm{ml}$ tubes and stored at $-20^{\circ} \mathrm{C}$. Serum biochemical indices, i.e., alanine aminotransferase (ALT), aspartate aminotransferase (AST), and alkaline phosphatase (ALP) activities; glucose (GLU), total protein (TP), and albumin (ALB) contents; lipid metabolism indices of total cholesterol (TC), triglycerides (TGs), low-density lipoprotein cholesterol (LDL-C) and high-density lipoprotein cholesterol (HDL-C) contents; and oxidant/antioxidant indices of malondialdehyde (MDA), total superoxide dismutase (T-SOD), glutathione peroxidase (GSH-Px), and catalase (CAT) concentrations, were determined using commercial kits (Nanjing Jiancheng Bioengineering Institute, Nanjing, Jiangsu, China). The serum cytokines, such as interleukin (IL)-1 $\beta$, IL-6, IL-10, and tumor necrosis factor- $\alpha$ (TNF- $\alpha$ ), were measured using the Multiskan MK3 Microplate Reader (Thermo Fisher Scientific, Wilmington, MA, USA). The serum concentrations of the immune indices, immunoglobulin (Ig)A, IgG, and IgM, were detected using the Hitachi 7600 Automatic Biochemical Analyzer.

\section{Gut Microbial Analysis}

At the end of the experiment, six hens per treatment were selected according to body weight and euthanized via sodium pentobarbital injection to obtain their cecal contents. The cecal contents were frozen in liquid nitrogen and stored at $-80^{\circ} \mathrm{C}$. Cecal microbial DNA was isolated using the Hexadecyl trimethyl ammonium bromide (CTAB)/Sodium dodecyl sulfate (SDS) method and quantified using the Qubit@2.0 Fluorometer (Thermo Fisher Scientific). The V4 region of the $16 \mathrm{~S}$ ribosomal RNA (16S rRNA) gene was then amplified with $515 \mathrm{~F}$ and $806 \mathrm{R}$ primers with the sequences of $5^{\prime}$-GTGCCAGCMGCCGCGGTAA-3' and $5^{\prime}$ GGACTACHVGGGTWTCTAAT-3'. DNA samples were quantified and then the V4 hypervariable region of the $16 \mathrm{~S}$ rDNA was amplified. The final amplicon pool was evaluated using the GeneJET ${ }^{\mathrm{TM}}$ Gel Extraction Kit (Thermo Fisher Scientific). Single-end reads were generated with the Ion S5 TM XL platform and filtered using the default parameters.

\section{Statistical Analysis}

Data analyses for performance, egg quality, and serum biochemical indices were performed using the Statistical Package for the Social Sciences (SPSS) version 19.0 for Windows (SPSS Incorporation, Chicago, Illinois, USA). Data were then analyzed using one-way ANOVA and means were compared using Duncan's multiple-range test. $p<0.05$ was considered as statistically significant. Data are expressed as the means and pooled SEM.

Single-end reads were assigned to samples based on their unique barcodes and truncated by cutting off the barcode and primer sequence. Raw reads were quality filtered under specific filtering conditions to obtain high-quality clean reads using Cutadapt (version 1.9.1). The reads were compared with the reference database using the UCHIME algorithm to detect chimera sequences, which were then removed to obtain the clean reads. Sequence analysis was performed using UPARSE software (version 7.0.1001). Sequences with $\geq 97 \%$ similarity were assigned to the same operational taxonomic units (OTUs). The subsequent clean reads were clustered as OTUs using UPARSE (version 7.0.1001) and annotated with the SILVA 16S rRNA gene database using the MOTHUR program (version 1.30.1). Alpha diversity (Shannon, Simpson, ACE, and Chaol richness indices) was calculated based on the OTU profiles from the MOTHUR program. Bar plots and heat maps were generated with the "vegan" package in R (version 2.15.3). Principal coordinate analysis (PCoA) was performed based on the Bray-Curtis distance using Quantitative Insights Into Microbial Ecology (QIIME) (version 1.9.1). Analysis of similarities was performed to compare the similarities of the bacterial communities among groups using the "vegan" package in R (version 2.15.3). A linear discriminant analysis (LDA) effect size (LEfSe) analysis was performed to identify the differentially enriched bacterial taxa in the different bacterial communities. Finally, the correlations between key parameters and bacterial communities were assessed via the Spearman's correlation analysis using the "pheatmap" package in $\mathrm{R}$ (version 2.15.3). Data are expressed as mean values.

\section{RESULTS}

\section{Performance and Egg Quality Parameters}

Table 2 lists the effects of dietary supplementation with PNE on the production performance of the laying hens. In the PNE100 group, the egg mass and FCR were significantly improved compared with those of the CONs $(p<0.05)$. The other performance indices differed among the groups, but not significantly $(p>0.05)$. Table 3 illustrates the egg quality parameters of hens supplemented with PNE. Dietary supplementation of 100 and $200 \mathrm{mg} / \mathrm{kg}$ PNE yielded higher yolk weights than those of the CON group $(p<0.05)$. No other egg quality parameters (i.e., shell thickness, shell strength, and Haugh units) differed among the groups at week 58 ( $p>0.05)$.

\section{Serum Biochemical and Lipid Metabolism Indices}

Figure 1 presents the effects of dietary PNE supplementation on serum biochemical indices. The PNE100 group had higher 
TABLE 2 | Effect of dietary pine needle extract (PNE) supplementation on production performance of laying hens ${ }^{1}(n=15)$.

\begin{tabular}{|c|c|c|c|c|c|c|}
\hline Treatment & CON & PNE100 & PNE200 & PNE400 & SEM & $P$-value \\
\hline $\mathrm{Fl}, \mathrm{g}$ & $101.75^{a b}$ & $100.30^{a}$ & $102.40^{b}$ & $102.38^{b}$ & 0.55 & 0.03 \\
\hline EP, \% & 82.81 & 90.36 & 88.21 & 86.68 & 2.16 & 0.14 \\
\hline EW, g & 58.10 & 58.49 & 57.40 & 58.93 & 0.73 & 0.75 \\
\hline EM, g & $48.08^{b}$ & $52.75^{\mathrm{a}}$ & $50.62^{a b}$ & $50.87^{a b}$ & 0.93 & 0.02 \\
\hline FCR, g/g & $2.07^{a}$ & $1.93^{b}$ & $2.00^{\mathrm{ab}}$ & $1.99^{a b}$ & 0.02 & 0.03 \\
\hline
\end{tabular}

${ }^{1} \mathrm{Fl}$, average daily feed intake; EP, egg production; EW, average egg weight; EM, daily egg mass; FCR, feed-to-egg ratio.

a,b Within a row, means with no common superscript differ significantly $(p<0.05)$.

TABLE 3 | Effect of dietary PNE supplementation on egg quality parameters of laying hens ${ }^{1}$.

\begin{tabular}{|c|c|c|c|c|c|c|}
\hline Treatment & CON & PNE100 & PNE200 & PNE400 & SEM & $P$-value \\
\hline Shell weight, $g$ & $10.38^{a b}$ & $10.21^{a}$ & $10.00^{a}$ & $10.80^{b}$ & 0.18 & 0.02 \\
\hline Yolk weight, g & $25.29^{a}$ & $26.69^{b}$ & $26.69^{b}$ & $25.99^{a b}$ & 0.03 & 0.02 \\
\hline Shell strength, N & 33.04 & 33.34 & 32.83 & 34.77 & 1.50 & 0.82 \\
\hline Shell thickness, $\mu \mathrm{m}$ & $430.00^{b}$ & $412.00^{\mathrm{ab}}$ & $390.00^{a}$ & $426.00^{b}$ & 0.01 & 0.03 \\
\hline Yolk color & 3.30 & 3.30 & 3.35 & 3.40 & 0.14 & 0.96 \\
\hline Haugh unit & $77.47^{\mathrm{bc}}$ & $79.09^{c}$ & $72.98^{a}$ & $75.53^{\mathrm{ab}}$ & 1.12 & 0.01 \\
\hline
\end{tabular}

a,b Within a row, means with no common superscript differ significantly $(p<0.05)$.

${ }^{1}$ Means were calculated using 15 replicates (two eggs per replicate) per treatment.

serum TP, ALB, and GLU concentrations than the CON group $(p<0.05)$. Compared with the CON group, ALT activity was decreased in the PNE100 group $(p<0.01)$. No other lipid metabolism indices (i.e., TC, TG, LDL-C, and HDL-C) differed (Figure 2).

\section{Serum Antioxidant, Cytokines, and Immune Indices}

Compared with the CON group, the MDA concentration was decreased $(p<0.05)$ and T-SOD, GSH-Px, and CAT activities were increased $(p<0.05)$ in the PNE100 group (Figure 3). Serum IL-1 $\beta$, IL-6, and TNF- $\alpha$ concentrations were reduced $(p<0.01)$, while the IL-10 concentration was increased in the PNE100 group ( $p<0.01$; Figure 4). Dietary PNE supplementation increased the $\operatorname{IgA}, \operatorname{IgG}$, and $\operatorname{IgM}$ concentrations in the PNE100 group $(p<0.01)$ compared with those of the CON group (Figure 5).

\section{Gut Microbial Diversity and Composition}

Bacterial alpha diversity in the cecal microbiota was estimated using the Shannon, Simpson, ACE, and Chaol indices of diversity and richness (Figures 6A-D). Compared with the CON group, the Shannon, ACE, and Chaol indexes were increased and the Simpson index was decreased in the PNE100 group, but insignificantly $(p>0.05)$, suggesting that supplementation of 100 $\mathrm{mg} / \mathrm{kg}$ PNE increased the overall bacterial richness of the cecal microbiota. Using PCoA based on the Bray-Curtis distance, discrimination levels were increased in the PNE100 group compared with those of the CON group $(p<0.10$; Figure 6E). Taxonomic compositions of the microbiotas were analyzed at the phylum and genus levels. Figure 6F shows the average relative abundances $(>1 \%)$ at the phylum level. Overall, the microbiotas were dominated by Bacteroidetes and Firmicutes, followed by Proteobacteria, Desulfobacterota, Actinobacteriota, Spirochaetota, Campilobacterota, Deferribacterota, and Euryarchaeota. Compared with the CON group, Proteobacteria was decreased notably in the PNE100 group, as the relative abundance of Bacteroidetes was increased and the relative abundance of Firmicutes was decreased. At the genus level (Figure 6G), the relative abundances of Vibrio, Lactobacillus, and Shewanella were decreased and the relative abundances of unclassified_o_Bacteroidale, unclassified_c_Bacteroidia Rikenellaceae_RC9_gut_group, unclassified_f_Rikenellaceae, norank_f_Eubacterium_coprostanoligenes_group, unclassified_ f_Lachnospiraceae, norank_f_norank_o_RF39, UCG-004, Helicobacter, and unclassified_f_Ruminococcaceae were increased in the PNE100 group compared with those of the CON group. Compared with those of the CON group, the relative abundances of Shewanella and Vibrio were significantly decreased and unclassified_f_Rikenellaceae, norank_ f_Eubacterium_coprostanoligenes_group, norank_f_norank_ o_Clostridia_UCG-014, norank_f_norank_o_RF39, norank_f Ruminococcaceae, Barnesiella, NK4A214_group, unclassified_f_ Tannerellaceae, Butyricicoccus, and Eisenbergiella were increased in the PNE100 group (Figure 6H).

\section{Relationship Between the Differential Bacterial Community and Main Parameters}

We performed the Spearman's correlation matrix to predict the correlation between the differential gut microbial 
A

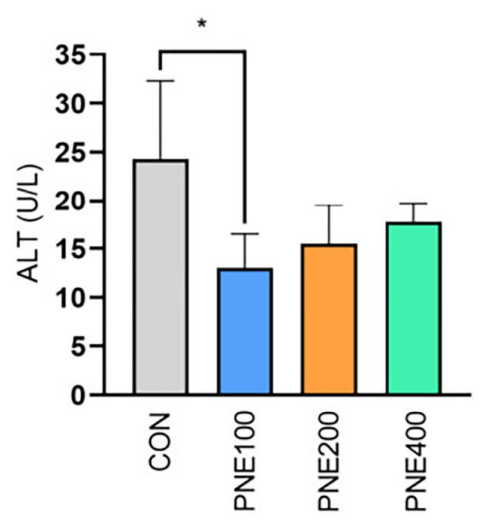

C
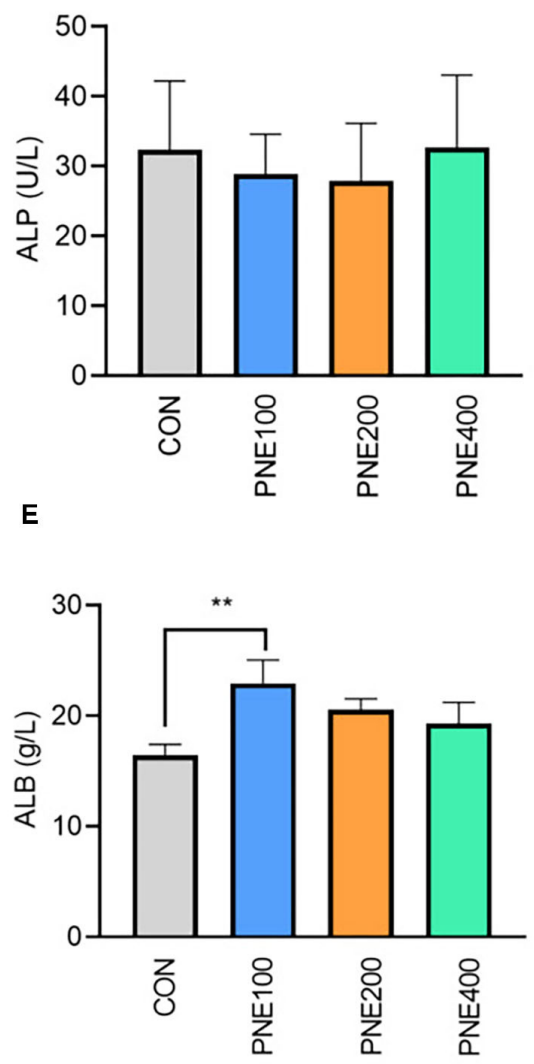

B

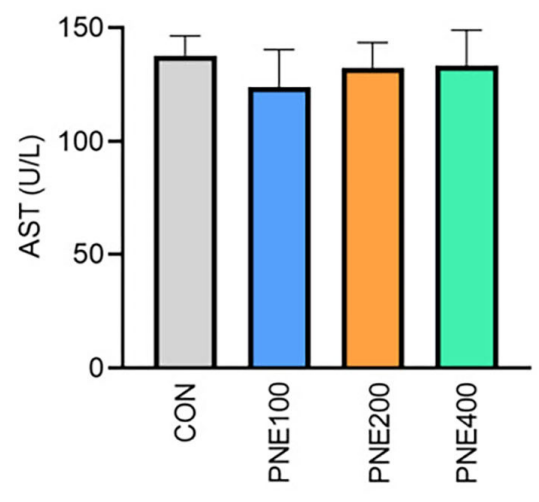

D
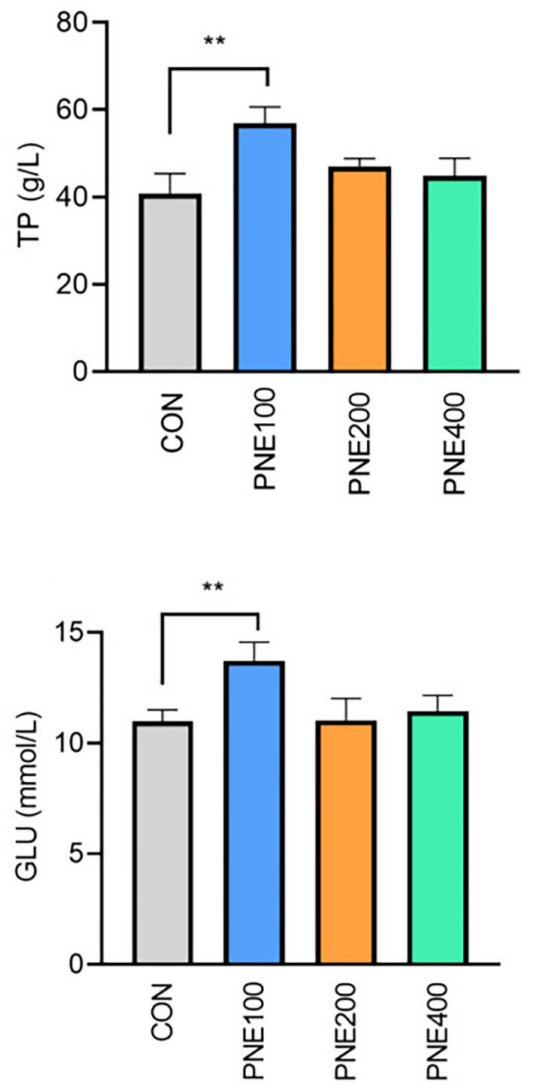

FIGURE 1 | Effect of pine needle extract (PNE) supplementation on serum biochemical indices ALT (A), AST (B), ALP (C), TP (D), ALB (E), and GLU (F) of laying hens. ALT, alanine aminotransferase; AST, aspartate aminotransferase; ALP, alkaline phosphatase; TP, total protein; ALB, albumin; GLU, glucose. Asterisks denote significant differences $\left({ }^{*} p \leq 0.05,{ }^{* *} p \leq 0.01\right), n=6$ per group, data are expressed as means \pm SD.

communities in their genera and key parameters. Egg mass and egg weight were positively correlated with the relative abundances of UCG-009 and Barnesiella and negatively correlated with the relative abundances of Vibrio and Shewanella (Figure 7A). Feed intake was positively correlated with Vibrio, Anaerobiospirillum, and norank_f_Ruminococcaceae and negatively correlated with Paraprevotella, UCG-009, and Angelakisella. The FCR was positively correlated with Vibrio and Shewanella and negatively correlated with the relative abundances of Paraprevotella, UCG-009, and Barnesiella. The Haugh units were positively correlated with norank_f_norank_o_RF39, unclassified_f_Rikenellaceae, 
A

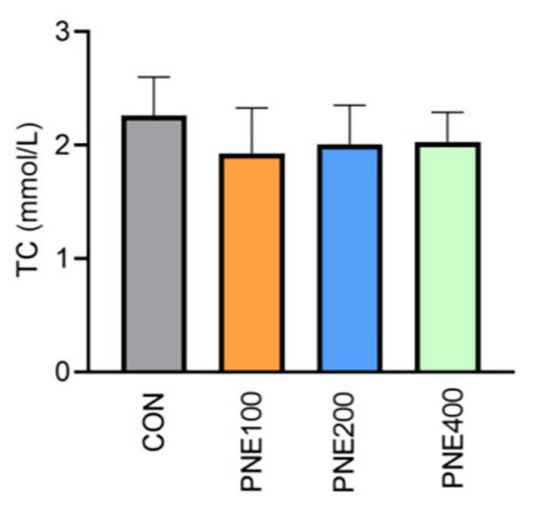

C

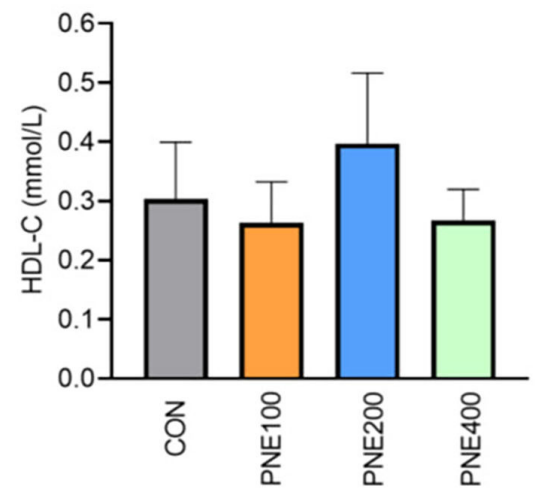

B

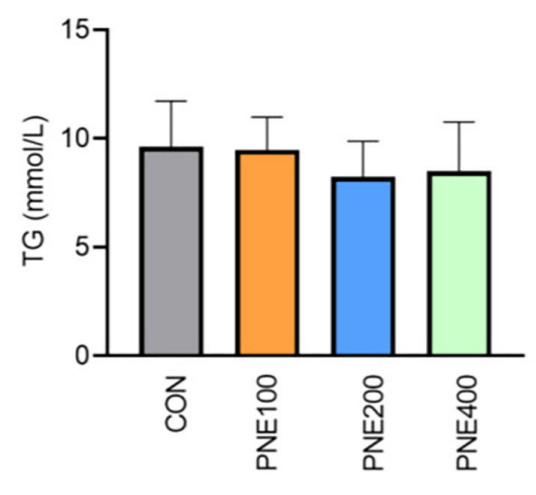

D

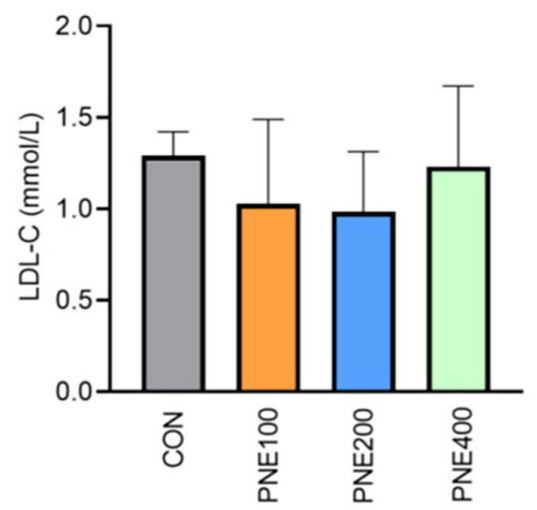

FIGURE 2 | Effect of pine needle extract (PNE) supplementation on serum lipid metabolism indices TC (A), TG (B), HDL-C (C), and LDL-C (D) of laying hens. TC, total cholesterol; TGs, triglycerides; HDL-C, high-density lipoprotein cholesterol; LDL-C, low-density lipoprotein cholesterol. $n=6$ per group, data are expressed as means $\pm \mathrm{SD}$.

and Oscillibacter and negatively correlated with Shewanella (Figure 7B). Yolk color was positively correlated with norank_ f_norank_o_RF39 and norank_f_norank_o_Clostridia_UCG014 and negatively correlated with the relative abundance of Fusobacterium. Yolk weight was positively correlated with norank_f_norank_o_Clostridia_UCG-014, norank_f_ Eubacterium_coprostanoligenes_group, and Family_XIII_ AD3011_group and negatively correlated with Shewanella. Shell color was positively correlated with Vibrio and Shewanella and negatively correlated with norank_f_norank_ o_Clostridia_UCG-014 and norank_f_norank_o_RF39. ALT was positively correlated with Shewanella and negatively correlated with norank_f_Eubacterium_coprostanoligenes_group, Family_XIII_AD3011_group, unclassified_f_Tannerellaceae, Angelakisella, unclassified_f_Rikenellaceae, NK4A214_ group, Oscillibacter, Eisenbergiella, Gallibacterium, and Butyricicoccus (Figure 7C). AST was positively correlated with Vibrio and Fusobacterium and negatively correlated with Barnesiella. TP was positively correlated with norank_f_ norank_o_Clostridia_UCG-014, Gallibacterium, Paraprevotella, UCG-009, and norank_f_norank_o_RF39 and negatively correlated with Vibrio, Shewanella, and Fusobacterium. ALB was positively correlated with norank_f_norank_o_ Clostridia_UCG-014, Gallibacterium, Paraprevotella, UCG-009, norank_f_norank_o_RF39, unclassified_f_Tannerellaceae, Angelakisella, and Family_XIII_AD3011_group and negatively correlated with the relative abundances of Vibrio, Shewanella, Fusobacterium, and Anaerobiospirillum. GLU was positively correlated with Gallibacterium, norank_f_Eubacterium coprostanoligenes_group, Paraprevotella, Barnesiella, and UCG-009 and negatively correlated with Anaerobiospirillum, Shewanella, Vibrio, and Fusobacterium. MDA was positively correlated with Anaerobiospirillum and negatively correlated with Butyricicoccus, Paraprevotella, and Gallibacterium. TSOD was positively correlated with norank_f_Eubacterium_ coprostanoligenes_group, Paraprevotella, Barnesiella, UCG-009, 


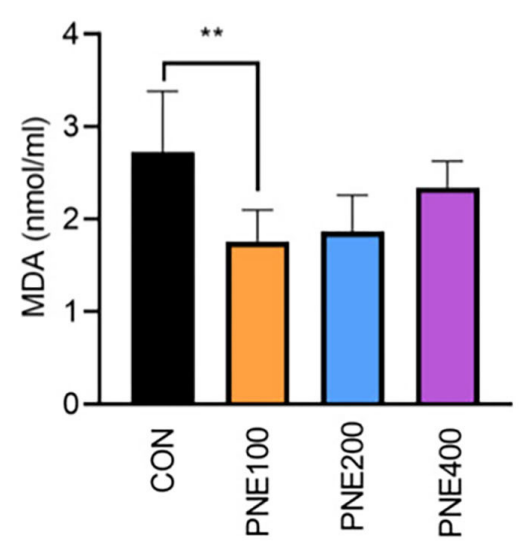

C

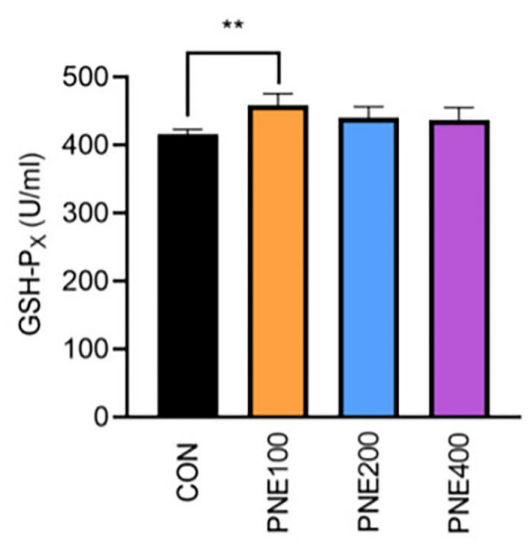

B

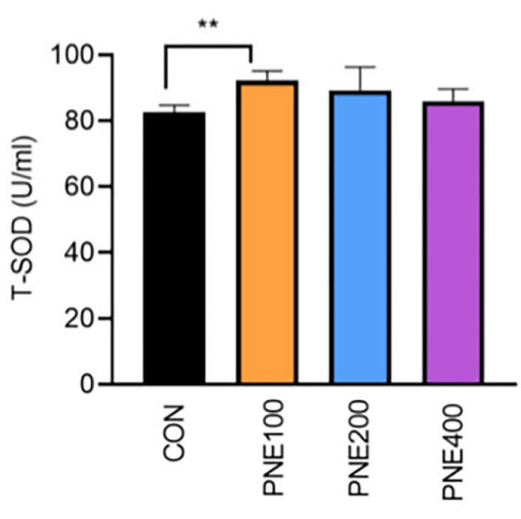

D

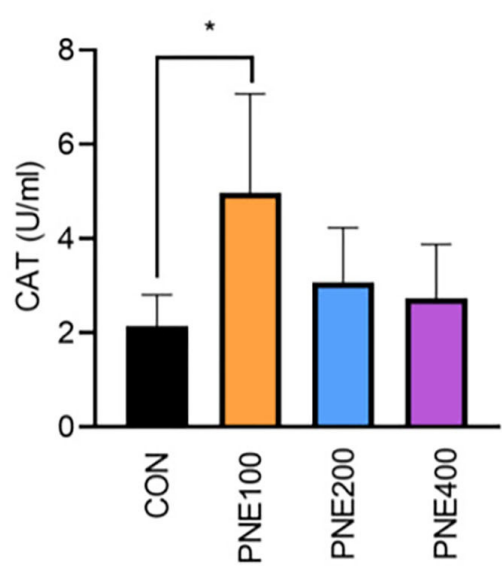

FIGURE 3 | Effect of pine needle extract (PNE) supplementation on serum antioxidant parameters MDA (A), T-SOD (B), GSH-PX (C), and CAT (D) of laying hens. MDA, malondialdehyde; T-SOD, total superoxide dismutase; GSH-Px, glutathione peroxidase; CAT, catalase. Asterisks denote significant differences $\left({ }^{\star} p \leq 0.05\right.$, ${ }^{\star \star} p \leq$ $0.01), n=6$ per group, data are expressed as means $\pm \mathrm{SD}$.

unclassified_f_Tannerellaceae, Angelakisella, Family_XIII_ AD3011_group, unclassified_f_Rikenellaceae, Oscillibacter, and NK4A214_group and negatively correlated with Shewanella, Anaerobiospirillum, and Vibrio. GSH-Px was positively correlated with norank_f_Eubacterium_coprostanoligenes_ group, Family_XIII_AD3011_group, unclassified_f_Rikenellaceae Gallibacterium, Butyricicoccus, Barnesiella, UCG-009, Oscillibacter, NK4A214_group, Eisenbergiella, unclassified_ $f \_$Tannerellaceae, Angelakisella, and norank_f_Ruminococcaceae and negatively correlated with Shewanella, Anaerobiospirillum, and Fusobacterium. CAT was positively correlated with Family_XIII_AD3011_group, Angelakisella, unclassified_ f_Rikenellaceae, Oscillibacter, NK4A214_group, norank_ f_Eubacterium_coprostanoligenes_group, Butyricicoccus, Paraprevotella, Barnesiella, and UCG-009 and negatively correlated with Shewanella. IL-6 was positively correlated with Anaerobiospirillum, Shewanella, and Vibrio and negatively correlated with Paraprevotella, Gallibacterium, norank $\_\_$
Eubacterium_coprostanoligenes_group, norank_f_norank_o_ Clostridia_UCG-014, and Butyricicoccus. IL-10 was positively correlated with Eisenbergiella, Gallibacterium, norank_ f_Eubacterium_coprostanoligenes_group, Butyricicoccus, NK4A214_group, Family_XIII_AD3011_group, norank_f_ Ruminococcaceae norank_f_norank_o_Clostridia_UCG-014, norank_f_norank_o_RF39_unclassified_f_Tannerellaceae, Angelakisella, unclassified_f_Rikenellaceae, and Oscillibacter and negatively correlated with Shewanella and Anaerobiospirillum. IL-1 $\beta$ was positively correlated with Anaerobiospirillum, Shewanella, and Vibrio and negatively correlated with Gallibacterium, norank_f_Eubacterium_coprostanoligenes_ group, Paraprevotella, Angelakisella, Family_XIII_AD3011_ group, unclassified_f_Rikenellaceae, Oscillibacter, and norank_f_norank_o_Clostridia_UCG-014. TNF- $\alpha$ was positively correlated with Anaerobiospirillum, Fusobacterium, and Shewanella and negatively correlated with norank_f_Eubacterium_ coprostanoligenes_group, Butyricicoccus, Paraprevotella, 
A

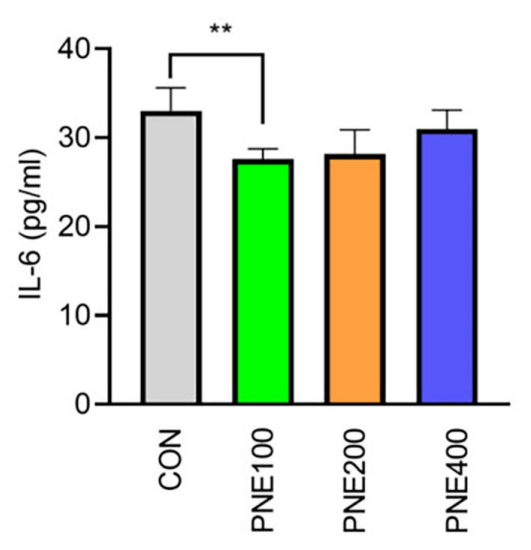

C

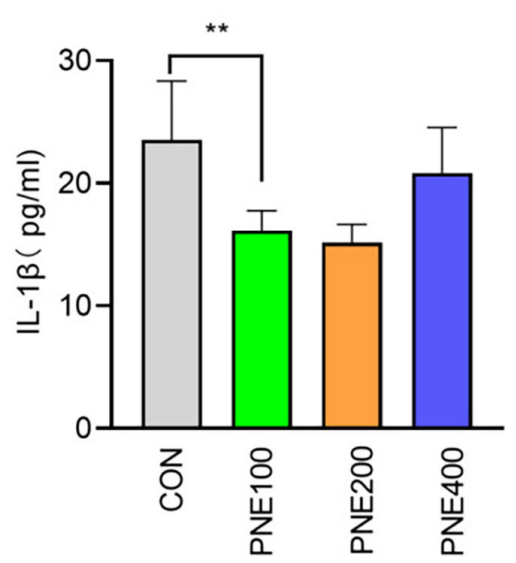

B

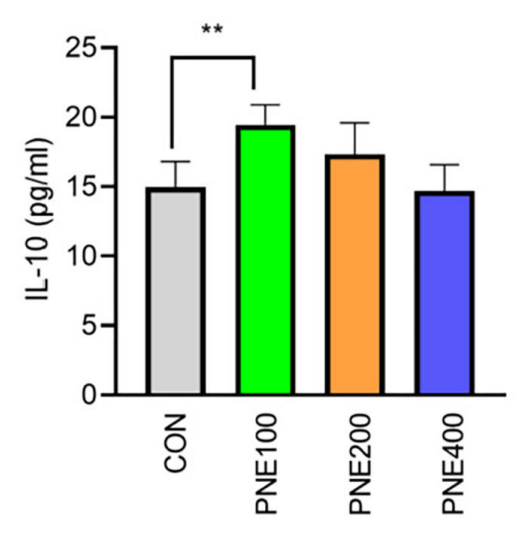

D

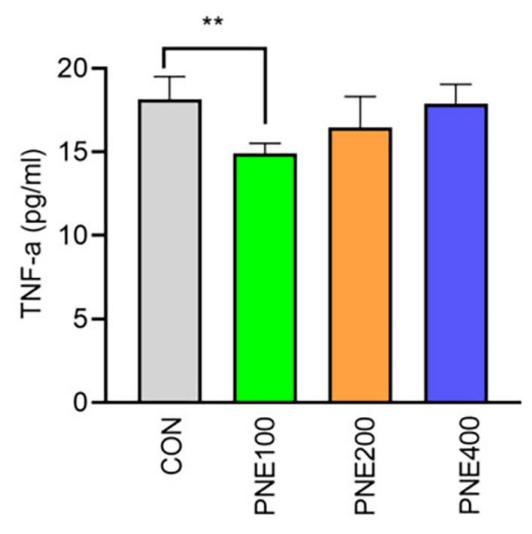

FIGURE 4 | Effect of pine needle extract (PNE) supplementation on serum inflammatory cytokines IL-6 (A), IL-10 (B), IL-1 $\beta$ (C), and TNF- $\alpha$ (D) of laying hens. IL-6, interleukin 6; IL-10, interleukin-10; IL-1 $\beta$, interleukin-1 $\beta$; TNF- $\alpha$, tumor necrosis factor- $\alpha$. Asterisks denote significant differences $\left({ }^{* *} p \leq 0.01\right), n=6$ per group, data are expressed as means $\pm \mathrm{SD}$.

Gallibacterium, Eisenbergiella, and norank_f_norank_ o_Clostridia_UCG-014. IgA was positively correlated with Gallibacterium, Paraprevotella, norank_f_norank_o_ Clostridia_UCG-014, norank_f_norank_o_RF39 unclassified_f_ Tannerellaceae, Angelakisella, UCG-009, norank_f_Eubacterium_ coprostanoligenes_group, and Butyricicoccus and negatively correlated with Shewanella, Vibrio, Anaerobiospirillum, and Fusobacterium. IgG was positively correlated with Gallibacterium, Paraprevotella, norank_f_norank_o_Clostridia_ UCG-014, norank_f_norank_o_RF39, UCG-009, and norank_f_ Eubacterium_coprostanoligenes_group and negatively correlated with Shewanella, Vibrio, Anaerobiospirillum, and Fusobacterium. IgM was positively correlated with Gallibacterium, norank_ f_norank_o_Clostridia_UCG-014, norank_f_norank_o_RF39, norank_f_Eubacterium_coprostanoligenes_group, Paraprevotella, and UCG-009 and negatively correlated with Shewanella, Vibrio, Anaerobiospirillum, and Fusobacterium.

\section{DISCUSSION}

Khan et al. reported that supplementation of $150 \mathrm{mg} / \mathrm{kg}$ PNE enhanced quail growth performance (16). Similarly, Kim et al. illustrated that broilers-fed $2.0 \%$ simple processed pine needle powder exhibited decreased overall body weight, average daily gain, average daily feed intake, and improved ileal protein digestibility compared with those of broilers-fed diets containing $0,0.5$, and $1.0 \%$ pine needle powder (13). Consistent with our results, Kothari et al. showed that adding 2.5 and $5 \mathrm{mg} / \mathrm{kg}$ fermented PNE increased egg mass and egg production (17). In this study, dietary supplementation of $100 \mathrm{mg} / \mathrm{kg}$ PNE to a cornsoybean meal-based diet improved both the FCR and egg mass of laying hens compared with those of the other groups during the trial period. Dietary supplementation of 100 or $200 \mathrm{mg} / \mathrm{kg}$ PNE also significantly improved the yolk weight. This may have been because the PNE promoted hepatic lipid metabolism by 


\section{A}

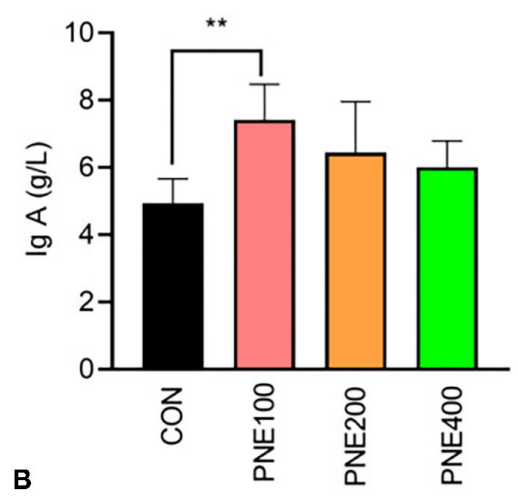

C
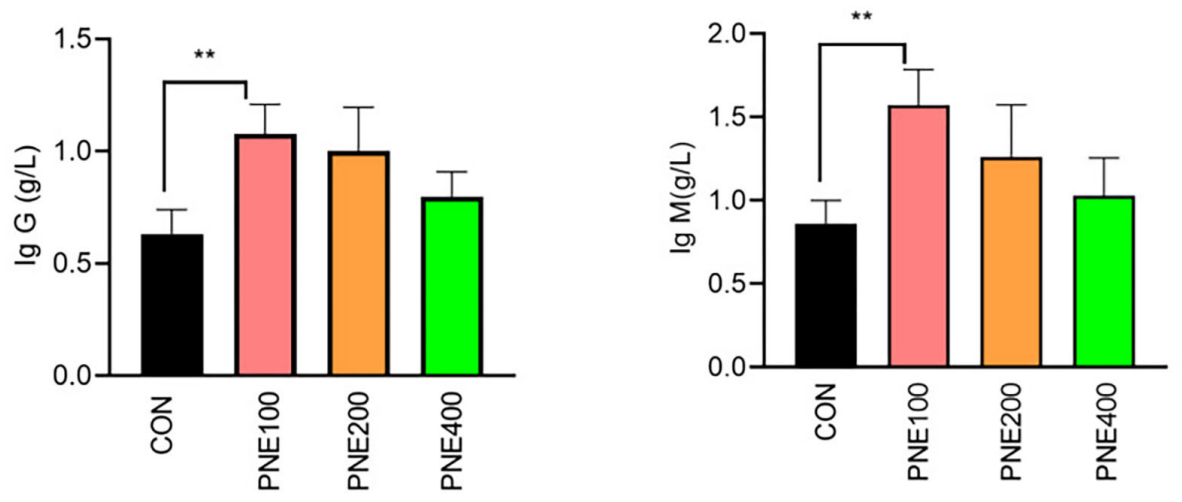

FIGURE 5 | Effect of pine needle extract (PNE) supplementation on serum immune parameters IgA (A), IgG (B), and IgM (C) of laying hens. IgA, immunoglobulin A; IgG, immunoglobulin G; IgM, immunoglobulin M. Asterisks denote significant differences ( $\left.{ }^{\star \star} p \leq 0.01\right)$, $n=6$ per group, data are expressed as means \pm SD.

protecting hepatocytes from oxidative damage, thus enhancing the yolk weight (19), which is consistent with the results of a previous study (20). The observed improvements in egg mass and feed efficiency after PNE supplementation might have been due to the presence of essential oils, terpenoids, and polyphenols, which are reported to improve nutrient digestion, absorption, and utilization in the digestive tract $(7,18)$.

Several serum indicators reflect animal health status. In healthy animals, protein synthesis increases along with TP and ALB. In our experiment, serum $\mathrm{TP}$ and ALB were higher in the PNE100 group than in the other groups, indicating a more vigorous protein metabolism in the PNE100 group, which was consistent with the results of Kim et al. (13), who found that $1 \%$ pine needle powder strengthened the ability of broilers to utilize protein and $\mathrm{N}$ nutrients. Khan et al. also demonstrated that including $150 \mathrm{mg} / \mathrm{kg}$ PNE ameliorated serum TP and ALB concentrations in quails (16). ALT and AST are important biomarkers for liver function and reflect individual body conditions. In this study, birds in the PNE100 group had lower serum ALT activity, indicating that PNE may have a liver protective function. Hens in the PNE100 group had relatively higher levels of glucose and abundances of Rikenellaceae_RC9_gut_group than the CON group. A previous study showed that Rikenellaceae_RC9_gut_group was positively correlated with serum insulin levels (21), which caused higher glucose.

In this study, lipid metabolism indices (e.g., HDL, LDL-C, TG, and TC) were significantly improved in the PNE100 group compared with those of the CON, PNE200, and PNE400 groups. This was consistent with previous findings that $0.6 \%$ pine needle powder more effectively decreased mortality and improved lipid peroxidation than 0.3 and $0.9 \%$ pine needle powder (22). The improved serum lipid indices herein were also consistent with those in rats as per Lee et al. (23), likely because (24) pine needles can decrease LDL oxidation and inflammatory actions by modulating Inducible nitric oxide synthase (iNOS) and Cyclooxygenase-2 (COX-2) expressions. Additionally, $0.6 \%$ pine needle powder inhibited lipid peroxidation when supplemented in the diets of broilers (25), which was similar to our results. Khan et al. also reported that adding $150 \mathrm{mg} / \mathrm{kg}$ PNE to quail diets decreased serum LDL-C, TG, and TC concentrations and increased serum HDL-C concentrations in quails. Therefore, our results suggest that $100 \mathrm{mg} / \mathrm{kg}$ PNE was suitable for ameliorating lipid peroxidation in laying hens.

Free radicals play important roles in immunity and signal transduction, but excessive free radicals can induce lipid 

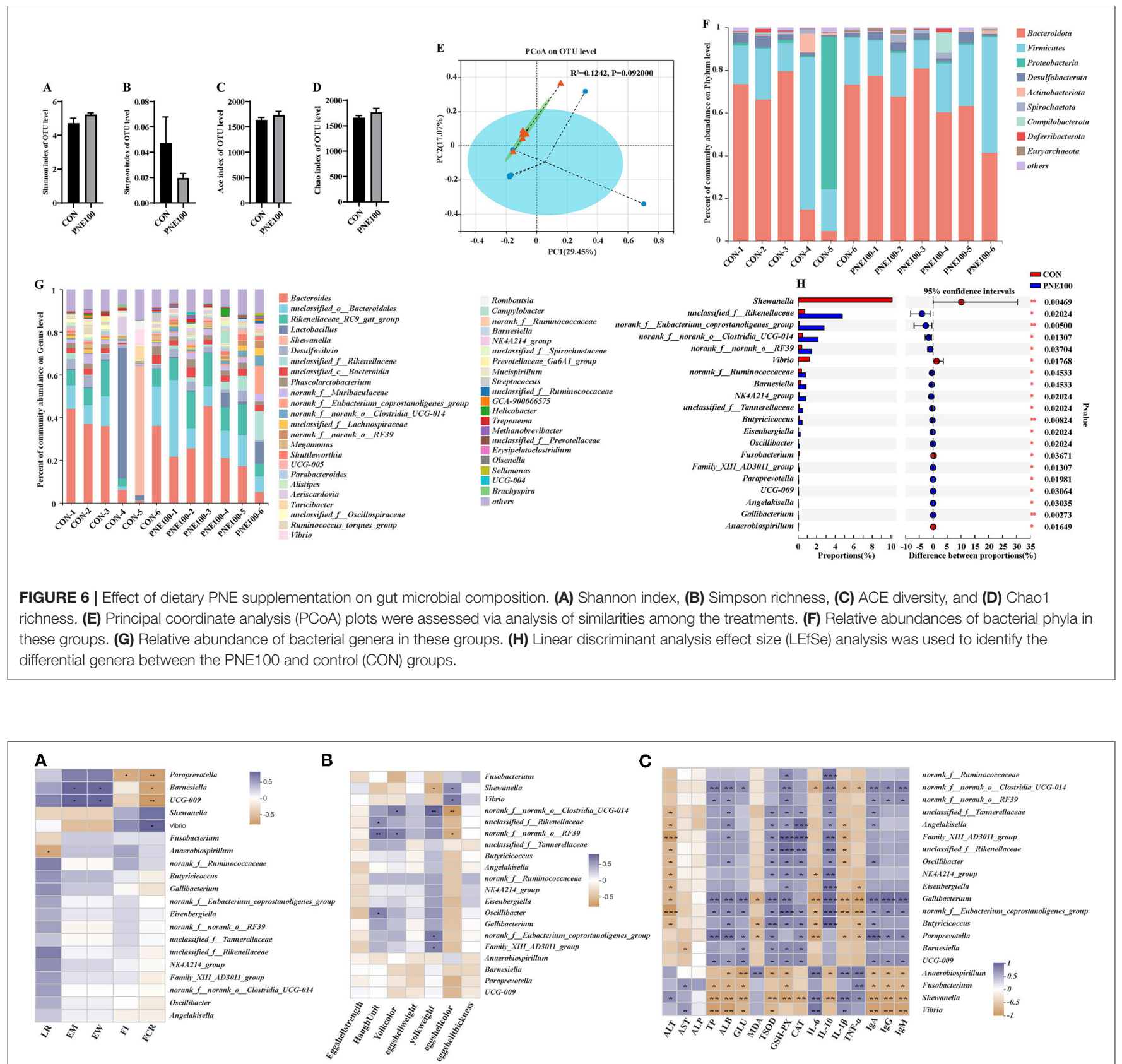

FIGURE 7 | Correlation between main parameters and differential microbes. (A) Relationship between the differential genera and performance. (B) Relationship between differential genera and egg quality parameters. (C) Relationship between differential genera and serum parameters. Asterisks denote significant differences $\left({ }^{*} p \leq 0.05,{ }^{* *} p \leq 0.01\right), n=6$ per group.

peroxidation in the cell membrane (26). Antioxidant enzymes such as T-SOD, GSH-Px, and CAT eliminate free radicals in the body (27); thus, antioxidant functions can be evaluated according to the antioxidant enzyme activities. Similarly, low MDA levels were shown to generate fewer free radicals in the body (28). Herein, supplementation with $100 \mathrm{mg} / \mathrm{kg}$ PNE yielded higher serum CAT, T-SOD, and GSH-Px activities and lower MDA concentrations, suggesting that $100 \mathrm{mg} / \mathrm{kg}$
PNE is suitable for elevating antioxidant functions in hens to reduce peroxide products. Chang et al. (29) found that rats fed $3 \mathrm{ml}$ PNE with proanthocyanidins had higher antioxidant abilities. Khan et al. reported that supplementation of $150 \mathrm{mg} / \mathrm{kg}$ PNE ameliorated the antioxidant status in quails by enhancing the activities of serum antioxidant enzymes, including GSHPx and T-SOD (16), which was consistent with our results. Kothari et al. demonstrated that 2.5 and $5 \mathrm{mg} / \mathrm{kg}$ fermented 
PNE improved the antioxidant status in broiler yolks (17), possibly because pine needles contain an abundance of active components, such as essential oils (30), proanthocyanidins (31), phenolics, and terpenoids (9), which are reported to have antioxidant abilities.

Ig G and IgM are important in anti-infection processes by engaging the phagocytic system and activating the complement system and IgA inhibits phagocytosis, chemotaxis, antibodydependent cellular cytotoxicity, and inflammatory cytokine release (35). PNE supplementation at $100 \mathrm{mg} / \mathrm{kg}$ significantly increased the serum IgG, IgM, and IgA concentrations compared with those of other groups, which was similar to the findings of Xiao et al., who reported that pine needle polysaccharides activated macrophages and enhanced the innate immune functions of broilers (36). Wei et al. showed that $0.1 \%$ pine needle powder ameliorated the immunity of chickens (10). However, this increase was not dose dependent because birds-fed diets containing 200 and $400 \mathrm{mg} / \mathrm{kg}$ PNE showed lower immune responses than the CON group. These data indicate that 100 $\mathrm{mg} / \mathrm{kg}$ PNE sufficiently induced high immune response levels in laying hens. Thus, we concluded that the improvements in egg mass and FCRs were best in the PNE100 group, likely owing to the presence of essential oils, terpenoids, and polyphenols in pine needles, which improved nutrient digestion, absorption, and utilization in the digestive tract (37).

Ig $G$ and IgM play important roles in anti-infection through engaging the phagocytic system and activating the complement system, while IgA can inhibit phagocytosis, chemotaxis, antibody-dependent cellular cytotoxicity, and the release of inflammatory cytokines (35). In this study, PNE supplementation at $100 \mathrm{mg} / \mathrm{kg}$ significantly increased the serum IgG, IgM, and IgA concentrations compared to the other groups, which was similar to Xiao et al. reported that pine needle polysaccharide activated macrophages and enhanced the innate immune function of broilers (36). In addition, Wei et al. showed that $0.1 \%$ of pine needle powder ameliorated the immunity of chickens (10). However, this increase did not obey dosedependent manners, when birds received the diets containing 200 and $400 \mathrm{mg} / \mathrm{kg}$ PNE showed lower immune response compared with the CON group. These data apparently indicated that 100 $\mathrm{mg} / \mathrm{kg}$ PNE was appropriate to induce a high level of immune response of laying hens. From the mentioned discussion, we can conclude that the observed improvements in egg mass and FCR of hens in the PNE100 group were the best, which might be due to the presence of essential oils, terpenoids, and polyphenols in pine needles improving digestion, absorption, and utilization of nutrients in the digestive tract (37).

Next, we examined the differential cecal microbes and their functions that may have contributed to the improved performance of the hens in the PNE100 group. Alpha and beta diversity analysis showed greater cecal bacterial richness and a significantly altered microbial composition. LEfSe analysis further identified the species that differed significantly between the PNE100 group and the other treatment groups. A more diverse gut microbial community is believed to positively affect the welfare and productivity of host birds (38). Bacteroidetes and Firmicutes were the dominant bacterial phyla, which together accounted for over $80 \%$ of the total microbial community detected in this study. The gut microecosystems of PNE-fed birds were exogenously altered at the phylum level and favored Bacteroidetes at expense of Firmicutes and Proteobacteria, thus leading to a lower Firmicutes/Bacteroidetes ratio. Studies in mice and humans have indicated that higher Firmicutes/Bacteroidetes ratios may play important roles in energy uptake (39). Higher Firmicutes/Bacteroidetes ratios are associated with host pathology (40) and the reverse is linked to healthy gut conditions (41). Another study found that the genus Bacteroides in the phylum Bacteroidetes was positively associated with the health status of broilers (42). Bacteroidetes comprises many bacteria that can digest complex substrates such as xylan (43) and cellulose (44), which produce propionate (45) and succinate (46), favoring intestinal nutrient absorption in the host. Proteobacteria contains opportunistic pathogens, such as Campylobacter, Escherichia, and Helicobacter, which have been found to be highly abundant in the feces of low-FCR birds $(47,48)$. Thereby, the increased abundance of Bacteroidetes along with the reduced abundances of Firmicutes and Proteobacteria might contribute to the ability of laying hens to effectively absorb and use nutrients. At the genus level, adding $100 \mathrm{mg} / \mathrm{kg}$ PNE reshaped the dominant gut bacterial species. In the PNE100 group, the relative abundances of unclassified_o_Bacteroidales, Rikenellaceae_RC9_gut_group, unclassified_f_Rikenellaceae, and Butyricicoccaceae were increased and the relative abundance of Lactobacillaceae was decreased. Lactobacillaceae, which is abundant in overweight or obese individuals, is positively correlated with serum High-sensitivity C-reactive protein (hsCRP) levels and occurs in the gut microbiotas of obese persons, suggesting low-grade inflammation (47). Rikenellalike bacteria use mucin as a carbon and energy source and are widespread in alimentary canals of animal (48); these bacteria benefit the diversity of the intestinal flora (49). Additionally, Rikenella is reported to help reduce endotoxemia markers (50). Butyricicoccaceae are involved in energy conversion providing intestinal energy to birds. Both of these bacteria can provide energy for the body and reduce inflammation and their increased abundances indicate host intestinal health. Therefore, the improved production performance of the PNE100 group in this study may be related to increased abundances of unclassified_o_Bacteroidales, Rikenella-like bacteria, and Butyricicoccaceae, which benefit the intestinal health of the host. These increased abundances of unclassified_o_Bacteroidales, Rikenella-like bacteria, and Butyricicoccaceae, along with the decreased abundance of Lactobacillaceae in the guts of the PNE100 group, may have contributed to the improved performance of hens.

We used correlation analysis to predict the relationship between the main parameters and the differential gut microbiota. In the PNE100 group, egg mass was positively correlated with Barnesiella, which can use fucosyllactose as an energy source in bacterial cultures to secrete linkage-specific fucosidase enzymes that free lactose to form an intestinal milieu that is resilient to inflammatory diseases (53). The FCR in the PNE100 group was positively correlated with Butyricicoccus, which can enhance feed conversion and protect broilers (54) from potentially 
harmful intestinal microorganisms and necrotic enteritis. Additionally, the Clostridial cluster IV strain of Butyricicoccus is a promising probiotic candidate for patients with inflammatory bowel disease (55). The yolk weight in the PNE100 group was positively correlated with norank_f_norank_o_Clostridia_UCG014, norank_f_Eubacterium_coprostanoligenes_group, and Family_XIII_AD3011_group. Studies have shown that Clostridium spp., a predominant cluster of commensal gut bacteria, exert beneficial effects on intestinal homeostasis and norank_f_norank_o_Clostridia_UCG-014 was demonstrated to have positive effects on mouse intestinal tracts (56). Additionally, norank_f_Eubacterium_coprostanoligenes_group has anti-inflammatory, antioxidant, and anticancer properties (57). Similarly, Family_XIII_AD3011_group has probiotic properties and improved intestinal health in weaning piglets after inoculation (56). We observed several correlations between various serum parameters and the hen gut microbiome. TP, ALB, GLU, T-SOD, GSH-Px, CAT, IL-10, IgA, IgG, and IgM were negatively correlated with the relative abundances of Vibrio, Shewanella, Fusobacterium, and Anaerobiospirillum and positively correlated with norank_f_norank_o_Clostridia_UCG-014, norank_f_Eubacterium_coprostanoligenes_group, Barnesiella, Paraprevotella, unclassified_f_Tannerellaceae, Angelakisella, Family_XIII_AD3011_group, unclassified_f_Rikenellaceae, Butyricicoccus, Barnesiella, Oscillibacter, and Eisenbergiella. Studies have illustrated that Shewanella is potentially pathogenic to humans and easily infects the intestinal tract (58). Vibrio is pathogenic to the gastrointestinal tract, particularly in immunocompromised individuals $(59,60)$. Fusobacterium was reported to easily impede colitis remission in mice (61). Anaerobiospirillum succiniciproducens is a rare cause of bacteremia in humans and can cause intestinal infection (62). Interestingly, some beneficial gut microbial communities were positively correlated with these serum parameters. For example, Clostridiaceae Family XIII AD3011 was shown to reduce inflammation levels and alter the gut microbiotas of healthy younger men (63). Oscillibacter produces valerate and enhances differentiation of IL-10-producing T-regulatory cells in vivo (64), which improves digestive tract function (65). Rikenellaceae is saccharolytic and ferments glucose to acid byproducts (66). Similarly, Parabacteroides is a beneficial bacterium that significantly increases serum IgA and butyric acid levels in animal digestive tracts (67). Eisenbergiella is an anaerobic bacterium in the Lachnospiraceae family that produces succinate and lactate and can elevate antioxidant levels in the liver, specifically those of T-SOD, CAT, and GSH-Px compared with CONs.

Serum ALT, MDA, IL-6, IL-1 $\beta$, and TNF- $\alpha$ are harmful indicators reflecting individual conditions. Here, these indicators were significantly lower in the PNE100 group than in the CON, PNE200, and PNE400 groups. These serum parameters were positively correlated with Anaerobiospirillum (62), Vibrio (59), Fusobacterium (61), and Shewanella (58), which are infectious pathogens. To the best of our knowledge, no study has reported the effects of pine needles on poultry intestinal flora, particularly in laying hens. However, previous reports suggest that pine ingredients such as essential oils have antibacterial properties; thus, we concluded that pine needles may have potential antibacterial effects and promote beneficial flora in the digestive tracts of hens. For example, pinosylvin (68) and essential oils (69) are components of pine that have been studied for their chemical compositions and have shown strong antimicrobial activity against common foodborne microorganisms (70). Hence, pine needles may potentially be used as natural antimicrobial agents (70). Further studies should focus on combining these effects for practical applications in laying hens. Our results showed that PNE promoted beneficial flora such as Butyricicoccus, Barnesiella, and Oscillibacter and inhibited harmful bacteria such as Fusobacterium and Anaerobiospirillum. Thus, we concluded that dietary supplementation of $100 \mathrm{mg} / \mathrm{kg}$ PNE triggered improvements in production performance and serum parameters regulated by the gut microbiome. In summary, alterations of the gut microbiome in the 100PNE group compared with those of the CON group suggested that $100 \mathrm{mg} / \mathrm{kg}$ PNE supplementation significantly modulated the cecal microbiota structure to more efficiently enhance protein utilization, protect liver function, improve lipid metabolism, boost antioxidant power, inhibit inflammatory factor expression, and increase immune responses, thus elevating production performance in laying hens.

\section{CONCLUSION}

Supplementing laying hen diets with $100 \mathrm{mg} / \mathrm{kg}$ PNE induced beneficial effects on the production performance in hens. The improved performance indices (i.e., egg mass, FCR, and yolk weight) contributed to altering the gut microbial community and structure. Additionally, hens-fed diets containing $100 \mathrm{mg} / \mathrm{kg}$ PNE had better liver function with lower ALT activity, better protein metabolism, and superior oxidant/antioxidant system functioning due to increased serum CAT, T-SOD, and GSHPx activities and decreased MDA. In summary, our results suggest that PNE supplementation at $100 \mathrm{mg} / \mathrm{kg}$ can improve the diversity and structure of the gut microbial composition, production performance, egg quality, and serum parameters in laying hens.

\section{DATA AVAILABILITY STATEMENT}

The original contributions presented in the study are included in the article/supplementary materials, further inquiries can be directed to the corresponding author/s.

\section{ETHICS STATEMENT}

The animal study was reviewed and approved by the Institutional Animal Care and Use Committee of China Agricultural University (Beijing, China), and the methods were carried out in accordance with the relevant guidelines and regulations. 


\section{AUTHOR CONTRIBUTIONS}

YG, SH, QM, and LZ designed the study. YG, SH, and QM conducted the experiments, drafted the manuscript, polished the manuscript, and finished the submission. YG, SH, JZ, and QM guided to analyze the experimental data, helped with revisiting, and reviewing the manuscript. All authors read and approved the final version of the manuscript.

\section{FUNDING}

This study was supported by the National Science Foundation of China (Grant No. 31930105), the special fund for

\section{REFERENCES}

1. Brisbin JT, Gong J, Sharif S. Interactions between commensal bacteria and the gut-associated immune system of the chicken. Animal Health Res Rev. (2008) 9:101-10. doi: 10.1017/S146625230800145X

2. Kers JG, Velkers FC, Fischer EAJ, Hermes GDA, Stegeman JA, Smidt H. Host and environmental factors affecting the intestinal microbiota in chickens. Front Microbiol. (2018) 9:235. doi: 10.3389/fmicb.2018.00235

3. Dong XY, Azzam MMM, Zou XT. Effects of dietary threonine supplementation on intestinal barrier function and gut microbiota of laying hens. Poultry Sci. (2017) 96:3654-63. doi: 10.3382/ps/pex185

4. Hussain T, Murtaza G, Kalhoro DH, Kalhoro MS, Metwally E, Chughtai MI, et al. Relationship between gut microbiota and host-metabolism: Emphasis on hormones related to reproductive function. Animal Nutr. (2021) 7:110. doi: 10.1016/j.aninu.2020.11.005

5. Scott KP, Gratz WS, Sheridan PO, Flint HJ, Duncan SH. The influence of diet on the gut microbiota. Pharmacol Res. (2013) 69:52. doi: 10.1016/j.phrs.2012.10.020

6. Yang Y, Iji PA, Choct M. Dietary modulation of gut microflora in broiler chickens: A review of the role of six kinds of alternatives to in-feed antibiotics. World's Poultry Sci J. (2009) 65:97-114. doi: 10.1017/S00439339090 00087

7. Windisch W, Schedle K, Plitzner CA. Use of phytogenic products as feed additives for swine and poultry. J Animal Sci. (2008) 86:E1408. doi: 10.2527/jas.2007-0459

8. Feng S, Zeng W, Luo F, Zhao J, Yang Z, Sun Q. Antibacterial activity of organic acids in aqueous extracts from pine needles (Pinus massoniana Lamb.). Food Sci. Biotechnol. (2010) 19:35-41. doi: 10.1007/s10068-010-0 005-2

9. Grassmann J, Hippeli S, Elstner EF. Plant's defence and its benefits for animals and medicine: Role of phenolics and terpenoids in avoiding oxygen stress. Plant Physiol. Biochem. (2002) 40:471-8. doi: 10.1016/S0981-9428(02)01395-5

10. Wei W, Hong-Ying $Z$, Xue-Bing W, Fang-Fang DU, Xiang-Yue DU, Fang L. Effect of pine needle on immune function and serum cholesterol of chickens. Animal Husbandry Feed Sci. (2014) 41:140-4. doi: 10.19578/j.cnki.ahfs.2015.04.012

11. Ohara A, Saito F, Matsuhisa T. Screening of antibacterial activities of edible plants against Streptococcus mutans. Food Sci Technol Res. (2008) 14:1903. doi: 10.3136/fstr.14.190

12. Kim YJ, Kim CM, Choi JH, Choi IH. Effect of dietary mugwort (Artemisia vulgaris L.) and pine needle powder (Pinus densiflora) on growth performance, serum cholesterol levels, and meat quality in broilers. J Biotechnol. (2012) 11:11866-73. doi: 10.5897/AJB11.3782

13. Kim E, Jung JY, Cho HM, Wickramasuriya SS, Maceline SP, Shin TK et al. Utilization of processed pine needle powder in broiler chicken diets. Poultry Sci. (2018) 45:167-74. doi: 10.5536/KJPS.2018.45.3.167

14. Kim YS, Shin DH. Volatile components and antibacterial effects of pine needle (Pinus densiflora S. And Z.) extracts. Food Microbiol. (2005) 22:3745. doi: 10.1016/j.fm.2004.05.002
China Agricultural Research System program (Grant No. CARS-40-K08), and the Special Fund from Chinese Universities Scientific Fund (2018TC043).

\section{ACKNOWLEDGMENTS}

We thank all the technicians in the experimental animal facility of China Agricultural University for providing daily care of laying hens. We also thank Shanghai Majorbio BioPharm Technology Corporation for their bioinformatic analysis of the data. We thank Traci Raley, MS, ELS, from Liwen Bianji (Edanz) (www.liwenbianji.cn/) for editing a draft of this manuscript.

15. Zeng W, Jia L. Antimicrobial activities of pine needle extracts. Food Sci. (2009) 30:87-90. doi: 10.1360/972009-754

16. Khan IU, Shah AA, Sahibzada FA, Hayyat A, Nazar M, Mobashar $\mathrm{M}$, et al. Carcass characteristics and serum biochemical profile of Japanese quail by the supplementation of pine needles and vitamin E powder. Biologia. (2019) 74:993-1000. doi: 10.2478/s11756-019-0 0225-y

17. Kothari D, Oh JS, Kim JH, Lee WD, Kim SK. Effect of dietary supplementation of fermented pine needle extract on productive performance, egg quality, and serum lipid parameters in laying hens. Animals (Basel). (2021) 11:1475. doi: 10.3390/ani110 51475

18. Murugesan GR, Syed B, Haldar S, Pender C. Phytogenic feed additives as an alternative to antibiotic growth promoters in broiler chickens. Front Vet Sci. (2015) 2:21. doi: 10.3389/fvets.2015. 00021

19. Bollengier-Lee S, Mitchell MA, Utomo DB, Williams PEV, Whitehead CC. Influence of high dietary vitamin E supplementation on egg production and plasma characteristics in hens subjected to heat stress. Br Poultry Sci. (1998) 39:106-12. doi: 10.1080/00071669889466

20. Lee JH, Kim KS, Shin SO. Effects of dietary pine cone meal on egg production, egg quality, serum cholesterol and cholesterol content and fatty acid composition of egg yolk in laying hens. Korean J Poultry Sci. (2007) 34:223-9. doi: 10.5536/KJPS.2007.34. 3.223

21. Zhou L, Xiao X, Zhang Q, Zheng J, Li M, Yu M, et al. Improved glucose and lipid metabolism in the early life of female offspring by maternal dietary genistein is associated with alterations in the gut microbiota. Front Endocrinol. (2018) 9:516. doi: 10.3389/fendo.2018. 00516

22. Park G, Paudyal MP, Park Y, Lee C, Hwang I, Tripathi GR, et al. Effects of pine needle extracts on plasma cholesterol, fibrinolysis and gastrointestinal motility. Biotechnol Bioprocess Eng. (2008) 13:2628. doi: 10.1007/s12257-008-0039-y

23. Lee E, Choi MY. Effects of pine needle on lipid composition and TBARS in rat fed high cholesterol. Korean J Food Sci Technol. (2000) 32:1186-90.

24. Yen G, Duh P, Huang D, Hsu C, Fu TY. Protective effect of pine (Pinus morrisonicola Hay.) needle on LDL oxidation and its anti-inflammatory action by modulation of NOS and COX-2 expression in LPS-stimulated RAW 264.7 macrophages. Food Chem Toxicol. (2008) 46:175-85. doi: 10.1016/j.fct.2007. 07.012

25. Kim Y. Effects of dietary supplementation of pine needle powder on carcass characteristics and blood cholesterol contents of broiler chicken. Korean J Poultry Sci. (2011) 38:51-7. doi: 10.5536/KJPS.2011.38. 1.051

26. Turner R, Baron T, Wolffram S, Minihane A M, Cassidy A, Rimbach G, et al. Effect of circulating forms of soy isoflavones on the oxidation of low density lipoprotein. Free Radical Res. (2004) 38:209-16. doi: 10.1080/10715760310001641854 
27. Kurata M, Suzuki M, Agar NS. Antioxidant systems and erythrocyte life-span in mammals. Comparat Biochem Physiol. (1993) 106:477-87. doi: 10.1016/0305-0491(93)9 0121-K

28. Lee E. Effects of powdered pine needle (Pinus densiflora seib et Zucc.) on serum and liver lipid composition and antioxidative capacity in rats fed high oxidized fat. J Korean Soc Food Sci Nutr. (2003) 32:92630. doi: $10.3746 / \mathrm{jkfn} .2003 .32 .6 .926$

29. Chang L, Xia G. Effect of pine needle extract on antioxidative ability of rats. Int J Front Med. (2020) 2:18-24. doi: 10.25236/IJFM.2020.020103

30. Aikaterini K, Samer T, Rouba S, Sofia L, Olga T, Vassilios R et al. Antioxidant potential of pine needles: A systematic study on the essential oils and extracts of 46 species of the genus pinus. Foods. (2021) 10:142. doi: 10.3390/foods 10010142

31. Park YS, Jeon MH, Hwang HJ, Park MR, Lee S, Kim SG, et al. Antioxidant activity and analysis of proanthocyanidins from pine (Pinus densiflora) needles. Nutr Res Practice. (2011) 5:281-7. doi: 10.4162/nrp.2011.5.4.281

32. Laskin DL, Sunil VR, Gardner CR, Laskin JD. Macrophages and tissue injury: agents of defense or destruction?. Ann Rev Pharmacol Toxicol. (2011) 51:267-88. doi: 10.1146/annurev.pharmtox.010909.105812

33. Chen W, Liu Y, Li M, Mao J, Zhang L, Huang R et al. Anti-tumor effect of $\alpha$ pinene on human hepatoma cell lines through inducing G2/M cell cycle arrest. J Pharmacol Sci. (2015) 127:332-8. doi: 10.1016/j.jphs.2015.01.008

34. Schulte W, Bernhagen J, Bucala R. Cytokines in sepsis: Potent immunoregulators and potential therapeutic Targets-An updated view. Media Inflam. (2013) 2013:e165974. doi: 10.1155/2013/165974

35. Andrew M, David M, Adams M, Ali K, Anderson R, Barnard D, et al. Venous thromboembolic complications (VTE) in children: First analyses of the Canadian Registry of VTE. Blood. (1994) 83:12517. doi: 10.1182/blood.V83.5.1251.1251

36. Xiaozhen C, Yan L, Tingting LI, Yu Y, Wenchao G, Kai Z, et al. Innate immunomodulatory effect of pine needle polysaccharide on chicken macrophage HD11. Sci Agri Sinica. (2020) 53:3180-6. doi: 10.3864/j.issn.0578-1752.2020.15.017

37. Sharma MK, Dinh T, Adhikari PA. Production performance, egg quality, and small intestine histomorphology of the laying hens supplemented with phytogenic feed additive. J Appl Poultry Res. (2020) 29:36271. doi: 10.1016/j.japr.2019.12.001

38. Janczyk P, Halle B, Souffrant WB. Microbial community composition of the crop and ceca contents of laying hens fed diets supplemented with Chlorella vulgaris. Poultry Sci. (2009) 88:2324-32. doi: 10.3382/ps.200900250

39. Ley RE, Turnbaugh PJ, Klein S, Gordon JI. Microbial ecologyHuman gut microbes associated with obesity. Nature. (2006) 444:1022-3. doi: 10.1038/4441022a

40. Turnbaugh PJ, Hamady M, Yatsunenko T, Cantarel BL, Duncan A, Ley $\mathrm{RE}$, et al. A core gut microbiome in obese and lean twins. Nature. (2009) 457:480-4. doi: 10.1038/nature07540

41. Ley RE, Bäckhed F, Turnbaugh P, Lozupone CA, Knight RD, Gordon JI. Obesity alters gut microbial ecology. PNAS. (2005) 102:11070-5. doi: 10.1073/pnas.0504978102

42. Zhang S, Zhong G, Shao D. Dietary supplementation with Bacillus subtilis promotes growth performance of broilers by altering the dominant microbial community. Poultry Sci. (2021) 100:100935. doi: 10.1016/j.psj.2020. 12.032

43. De Filippo C, Cavalieri D, Di Paola M, Ramazzotti M, Poullet JB, Massart $\mathrm{S}$, et al. Impact of diet in shaping gut microbiota revealed by a comparative study in children from Europe and rural Africa. PNAS. (2010) 107:146916. doi: 10.1073/pnas.1005963107

44. Wang K, Liao M, Zhou N, Bao L, Ma K, Zheng Z, et al. Parabacteroides distasonis alleviates obesity and metabolic dysfunctions via production of succinate and secondary bile acids. Cell Rep. (2019) 26:222. doi: 10.1016/j.celrep.2018. 12.028

45. Craven M, Egan CE, Dowd SE, Mcdonough SP, Dogan B, Denkers $\mathrm{EY}$, et al. Inflammation drives dysbiosis and bacterial invasion in murine models of ileal crohn's disease. PLoS ONE. (2012) 7:e41594. doi: 10.1371/journal.pone.0041594
46. Dey N, Soergel D A W, Repo S, Brenner S E. Association of gut microbiota with post-operative clinical course in Crohn's disease. BMC Gastroenterol. (2013) 13:131. doi: 10.1186/1471-230X-13-131

47. Manz W, Amann R, Ludwig W, Wagner M, Schleifer KH. Phylogenetic oligodeoxynucleotide probes for the major subclasses of proteobacteria: Problems and solutions. Syste Appl Microbiol. (1992) 15:593-600. doi: 10.1016/S0723-2020(11)80121-9

48. Mukhopadhya I, Hansen R, El-Omar E. IBD-what role do Proteobacteria play?. Nat Rev Gastroenterol Hepatol. (2012) 9:219-30. doi: 10.1038/nrgastro.2012.14

49. Bervoets L, Van Hoorenbeeck K, Kortleven I, Van Noten C, Hens $\mathrm{N}$, Vael $\mathrm{C}$, et al. Differences in gut microbiota composition between obese and lean children: A cross-sectional study. Gut Pathogens. (2013) 5:10. doi: $10.1186 / 1757-4749-5-10$

50. Woese CR, Kandler O, Wheelis ML. Towards a natural system of organisms: proposal for the domains Archaea, Bacteria, and Eucarya. Proc Nat Acad Sci. (1990) 87:4576-9. doi: 10.1073/pnas.87.12.4576

51. Oki K, Toyama M, Banno T, Chonan O, Benno Y, Watanabe K. Comprehensive analysis of the fecal microbiota of healthy Japanese adults reveals a new bacterial lineage associated with a phenotype characterized by a high frequency of bowel movements and a lean body type. BMC Microbiol. (2016) 16:284. doi: 10.1186/s12866-016-0898-x

52. Yang $J$, Zhang $S$, Henning $S \mathrm{M}$, Lee $\mathrm{R}$, Hsu $\mathrm{M}$, Grojean $\mathrm{E}$, et al. Cholesterol-lowering effects of dietary pomegranate extract and inulin in mice fed an obesogenic diet. J Nutr Biochem. (2018) 52:3. doi: 10.1016/j.jnutbio.2017.10.003

53. Daillere R, Vetizou M, Waldschmitt N, Yamazaki T, Isnard C, PoirierColame V et al. Enterococcus hirae and Barnesiella intestinihominis facilitate cyclophosphamide-induced therapeutic immunomodulatory effects. Immunity. (2016) 45:931-43. doi: 10.1016/j.immuni.2016. 09.009

54. Keerqin C, Rhayat L, Zhang ZH, Gharib-Naseri K, Kheravii SK, Devillard E, et al. Probiotic Bacillus subtilis 29,784 improved weight gain and enhanced gut health status of broilers under necrotic enteritis condition. Poultry Sci. (2021) 100:4. doi: 10.1016/j.psj.2021. 01.004

55. Geirnaert A, Steyaert A, Eeckhaut V. Butyricicoccus pullicaecorum, a butyrate producer with probiotic potential, is intrinsically tolerant to stomach and small intestine conditions. Anaerobe. (2014) 30:70-4. doi: 10.1016/j.anaerobe.2014. 08.010

56. Yu S, Zhang G, Liu Z, Wu P, Yu Z, Wang J. Repeated inoculation with fresh rumen fluid before or during weaning modulates the microbiota composition and co-occurrence of the rumen and colon of lambs. BMC Microbiol. (2020) 20:1716. doi: 10.1186/s12866-020-1 716-z

57. Li L, Buhman KK, Hartman PA, Beitz DC. Hypocholesterolemic effect of eubacterium coprostanoligenes atcc-51222 in rabbits. Lett Appl Microbiol. (1995) 20:137-40. doi: 10.1111/j.1472-765X.1995.tb0 0410.x

58. Holt HM, Gahrn-Hansen B, Bruun B. Shewanella algae and Shewanella putrefaciens: Clinical and microbiological characteristics. Clin Microbiol Infection. (2005) 11:347-52. doi: 10.1111/j.1469-0691.2005. 01108.x

59. Austin B, Zhang XH. Vibrio harveyi: A significant pathogen of marine vertebrates and invertebrates. Lett Appl Microbiol. (2006) 43:119-24. doi: 10.1111/j.1472-765X.2006.01989.x

60. Amaro C, Biosca EG. Vibrio vulnificus biotype 2, pathogenic for eels, is also an opportunistic pathogen for humans. Appl Environ Microbiol. (1996) 62:1454-7. doi: 10.1128/aem.62.4.1454-1457.1996

61. Yamashita T, Tai S, Tsukahara T, Inoue R. Fusobacterium nucleatum impedes remission of colitis in a mouse model. Biosci Biotechnol Biochem. (2021) 85:1235-42. doi: 10.1093/bbb/zbab029

62. Pienaar C, Kruger AJ, Venter EC, Pitout JD. Anaerobiospirillum succiniciproducens bacteraemia. J Clin Pathol. (2003) 56:3168. doi: $10.1136 /$ jcp. 56.4 .316

63. Burton KJ, Rosikiewicz M, Pimentel G, Butikofer U, von Ah U, Voirol $\mathrm{M}$, et al. Probiotic yogurt and acidified milk similarly reduce postprandial 
inflammation and both alter the gut microbiota of healthy, young men. $\mathrm{Br}$ J Nutr. (2017) 117:1312-22. doi: 10.1017/S0007114517000885

64. Li J, Sung CYJ, Lee N, Ni Y, Pihlajamaki J, Panagiotou G, et al. Probiotics modulated gut microbiota suppresses hepatocellular carcinoma growth in mice. Proc Natl Acad Sci USA. (2016) 113:E1306-15. doi: 10.1073/pnas.1518189113

65. Sidira M, Kandylis P, Kanellaki M, Kourkoutas Y. Effect of curing salts and probiotic cultures on the evolution of flavor compounds in dry-fermented sausages during ripening. Food Chem. (2016) 201:84. doi: 10.1016/j.foodchem.2016. 01.084

66. Mavromatis K, Stackebrandt E, Munk C, Lapidus A, Nolan M, Lucas S, et al. Complete genome sequence of the bile-resistant pigment-producing anaerobe Alistipes finegoldii type strain (AHN2437(T)). Stand Genom Sci. (2013) 8:26-36. doi: 10.4056/sigs.35 27032

67. Guo C, Cui Q, Cheng J, Chen J, Zhao Z, Guo R et al. Probioticfermented Chinese dwarf cherry Cerasus humilis (Bge.) Sok. Juice modulates the intestinal mucosal barrier and increases the abundance of Akkermansia in the gut in association with polyphenols. J Funct Foods. (2021) 80:104424. doi: 10.1016/j.jff.2021.1 04424

68. Lee SK, Lee HJ, Min HY, Park EJ, Lee KM, Ahn YH, et al. Antibacterial and antifungal activity of pinosylvin, a constituent of pine. Fitoterapia. (2005) 76:258-60. doi: 10.1016/j.fitote.2004.12.004
69. Kartnig T, Still F, Reinthaler F. Antimicrobial activity of the essential oil of young pine shoots (Picea abies L.). J Ethnopharmacol. (1991) 35:1557. doi: $10.1016 / 0378-8741(91) 90067-\mathrm{N}$

70. Zeng W C, Zhang Z, Gao H. Chemical composition, antioxidant, and antimicrobial activities of essential oil from pine needle (Cedrus deodara). $J$ Food Sci. (2012) 77:C824-9. doi: 10.1111/j.1750-3841.2012.02767.x

Conflict of Interest: The authors declare that the research was conducted in the absence of any commercial or financial relationships that could be construed as a potential conflict of interest.

Publisher's Note: All claims expressed in this article are solely those of the authors and do not necessarily represent those of their affiliated organizations, or those of the publisher, the editors and the reviewers. Any product that may be evaluated in this article, or claim that may be made by its manufacturer, is not guaranteed or endorsed by the publisher.

Copyright $\odot 2022$ Guo, Huang, Zhao, Zhang, Ji and Ma. This is an open-access article distributed under the terms of the Creative Commons Attribution License (CC $B Y)$. The use, distribution or reproduction in other forums is permitted, provided the original author(s) and the copyright owner(s) are credited and that the original publication in this journal is cited, in accordance with accepted academic practice. No use, distribution or reproduction is permitted which does not comply with these terms. 